\title{
Ligand-based Drug Design Approach in Designing New Leads from Existing Pharmacophores; Binding Pocket Estimation and Binding Pocket-directed Screening of Antiviral Drugs for Treatment of Novel Corona COVID-19 and Topoisomerase Inhibitors
}

\author{
Dushyanth R Vennapu ${ }^{1}$, Mahesh S Palled ${ }^{2}$
}

\begin{abstract}
Using the homology modeling-derived protein structure, and literature-reported cases of the treatment of coronavirus by chloroquine and remdesivir. ICMR guidelines for the usage of a low toxic metabolite of chloroquine hydroxychloroquine as prophylactic medication for healthcare professionals as preventive measures for those who treat corona patients. Further, Japanese flu drug favipiravir in treatment is efficient in the treatment of corona. The protein model suggested by I-TASSER was used in docking; the active site of the protein was predicted using similarity search and the potential leads are screened for their efficiency in combating corona outbreak from epidemic to pandemic.

Keywords: Docking, Hits and leads, Homology.

Indian Journal of Medical Biochemistry (2020): 10.5005/jp-journals-10054-0160
\end{abstract}

\section{INTRODUCTION}

Coronavirus outbreak started in Wuhan city of China, on December 31,2019; the same was renamed as coronavirus 2019 (COVID-19) (2019 novel coronavirus) by World Health Organization and almost spread globally affecting $>94$ countries with confirmed cases of 416,686 and reported deaths about 18,589 (as on $26 / 03 / 2020$ ). The major source of contamination is through droplets from the infected person to a healthy person similar to all flu or respiratory infections. Selfsanitation is the only method to protect self and clinical trial evidence suggests the use of chloroquine and remdesivir for treatment.

\section{Spillover-based Classification of Corona-COVID-19}

The transmission of the virus from animals to humans is termed spillover. In India with respect to Kerala state, the outbreak of Nipah virus is an example of spillover from bats, the transmission may be either lead by a mutation in the virus or by increased contacts with pets or dead animals. Similarly, the MERS-COV transmission is from camels and SARS-COV from bats. The exact source of transmission of the 2019 novel coronavirus-COVID-19 is unknown.

Sporadic level: Occasional cases: Occurring at irregular intervals, Endemic level: Persistent occurrence with a low to moderate level, Hyperendemic level: Occurrence clearly in excess of the expected level for a given period, Pandemic: Epidemic spread over several countries or continents affecting a large number of people. Epicenter: WHO declared Europe as an Epicenter for corona outbreak in India Maharashtra, especially Mumbai and Pune are the epicenters for the outbreak. The coronavirus symptoms or effects are classified based on the pathology as mild or severe infections mild cases include common cold to pneumonia severe cases include severe acute reparatory syndrome coronavirus first reported in China-2003 and Middle East respiratory syndrome reported in Saudi Arabia in 2012. Polymerase chain reaction (PCR) is the only known method of Diagnosis National Institute of Virology
'Department of Pharmaceutical Chemistry, KLE University College of Pharmacy, Belagavi, Karnataka, India; Department of Chemistry, Central University of Punjab, Bathinda, Punjab, India

${ }^{2}$ Department of Pharmaceutical Chemistry, KLE University College of Pharmacy, Belagavi, Karnataka, India

Corresponding Author: Dushyanth R Vennapu, Department of Pharmaceutical Chemistry, KLE University College of Pharmacy, Belagavi, Karnataka, India; Department of Chemistry, Central University of Punjab, Bathinda, Punjab, India, Phone: +91 8919754133 , e-mail: dushyanthreddy233@gmail.com

How to cite this article: Vennapu DR, Palled MS. Ligand-based Drug Design Approach in Designing New Leads from Existing Pharmacophores; Binding Pocket Estimation and Binding Pocketdirected Screening of Antiviral Drugs for Treatment of Novel Corona COVID-19 and Topoisomerase Inhibitors. Indian J Med Biochem 2020;24(3):143-148.

Source of support: KLE University and Central University of Punjab Conflict of interest: None

is the only authorized center for analysis. Neither the treatment nor the vaccine yet available are underway.

The phylogenetic and whole-genome analysis of literature ${ }^{1}$ suggests that close homology between novel coronavirus-COVID-19 with Batcov TaTG13 detected in bats from Yunnan Province. ${ }^{2}$

As the clinical trials and in vivo, testing suggests that these two molecules chloroquine and remdesivir would effective in combating the coronavirus. The current research focused on docking the chloroquine and remdesivir onto the predicted pockets and reengineering the existing drugs as newer leads in combating novel coronavirus-COVID-19. The model protein based on the genome sequences deposited in the literature and as suggested by I-TASSER the protein model (QHD43415) used in docking studies. 


\section{Topology Modeling and Binding Site Prediction}

The literature report suggested that the phylogenic similarity between novel coronavirus 19 and batcov. Batcov protein retrieved from the protein data bank with PDB ID:5GXR, using the active site model of 5GXR performed by motif search option by MEGA and genome sequence database a model protein (QHD43415) was retrieved from I-TASSER with TM-Score 0.55 and analyzed for RMSD values using RAMPAGE for novel coronavirus-19. Qiagens CLC drug Discovery Workbench 4.0 deployed in predicting the binding site. CLC workbench suggested six binding pockets; all the binding pockets were screened using chloroquine and remdesivir as potential leads. Further hydrogen bonding interaction was taken as one of the criteria in screening the binding pockets (Figs 1 and 2).

Most literature reported case history of recovered patents across the globe utilized the nucleotide analogs for the treatment of viral infection of novel corona-COVID-19. Symptomatic treatment is effective with chloroquine and other retroviral drugs and even with the swine flu medication oseltamivir (Fig. 3). ${ }^{3-5}$

Active site engineering of protein model of novel coronavirus 2019 (QHD43415) using favipiravir (Fig. 4).

Active site engineering of protein model of novel coronavirus 2019 (QHD43415) using chloroquine and remdesivir (Figs 5 to 8).

\begin{tabular}{llll}
\hline Ligand & Protein & Interacting residues & Dock score \\
\hline Chloroquine & QHD43415 & $\begin{array}{l}\text { LEU88, GLU57, ARG24, } \\
\text { TYR154, and GLU87 }\end{array}$ & -66.67 \\
Remdesivir & QHD43415 & $\begin{array}{l}\text { TYR154, GLU10, and } \\
\text { LEU88 }\end{array}$ & -44.47 \\
Favipiravir & QHD43415 & LEU88 and GLU155 & -28.67 \\
KAL-03 & QHD43415 & $\begin{array}{l}\text { TYR154, LYS125, and } \\
\text { LEU88 }\end{array}$ & -56.50 \\
& & TYR154 and LYS125 & --48.11 \\
\hline
\end{tabular}

Other molecules of the KAL series shown interaction with TYR154 only The proposed binding site might contain the residues LEU88, TYR154, GLU87, LEU88, and GLU10

The common pharmacophore of chloroquine and remdesivir directed that interacting residues would play a major role in

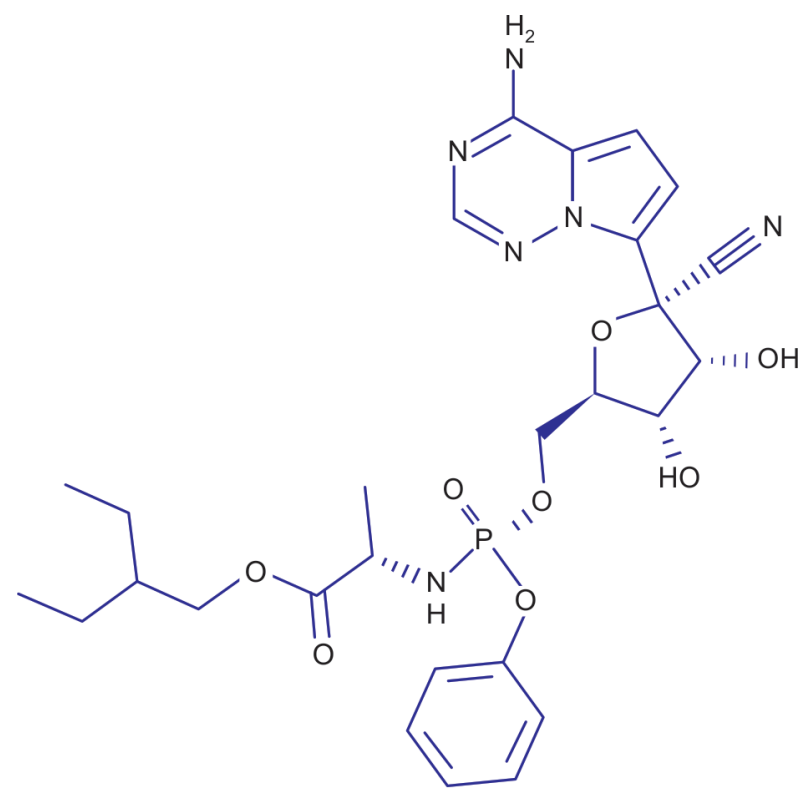

Fig. 1: Remdesevir the treatment of corona. Our recent molecules quinoline-based hydrazones as anticancer and antimicrobial published from our lab, due to structural similarities molecules were screened against the protein model of novel coronavirus. The quinolinebased hydrazones were screened on cancer cell lines and for their antimicrobial studies. Further, the drug-likeness and toxicity profiling of the same were estimated by molecular properties protocol of CLC and FAFdrugs4 webserver (Fig. 9).

\section{Ligand-based Pharmacophore Modeling: QSAR Approach in Screening Ligands on Modeled Active Site}

E-pharmacophore was designed using remdesivir and chloroquine as the exact protein structure and the active site was not elucidated yet. The pharmacophore model was screened with all FDAapproved antimalarial drugs as well as reported molecules from our lab. The spike proteins of novel coronavirus COVID-19 are reported to be interacting with angiotensin-converting enzyme (ACE). The clinical symptoms of corona are subsided upon treatment with chloroquine. The ligands remdesivir chloroquine and quinolinebased hydrazones and reengineered ligand were docked on the predicted active site of batcov protein and a model protein provided by I-TASSER (Fig. 10). ${ }^{6}$

\section{Drug-likeness of KAL Series and Toxicity Prediction}

The molecules were screened virtually for their drug-likeness and estimated for ADMET properties virtually on the FAFdrugs4 webserver. All the molecules obey mostly Lipinski parameters except with MolLogP data (Figs 11 and 12).

\section{Site-specific or Active Site Docking on TOPO-2 (1ZXM)}

Novel quinoline-based hydrazones were screened against cancer cell lines, the molecules were evaluated virtually for their ADMET properties using the FAF webserver/SWIS ADME webserver. In continuation to our research in ascertaining anticancer activity, we also focused to identify whether the reported molecules are selective to both Topo-1 and Topo-2 or specifically to either of these by virtual docking. The versatility of screening molecules and converting hits to leads play a vital role in drug design.

All the ligands were sketched using Chemdraw-Ultra-12, the 2D molecules were converted into 3D by Chem3Dpro-12, the molecules were energy minimized using MM2, the energy minimized molecules were imported into the CLC Drug Discovery workbench.

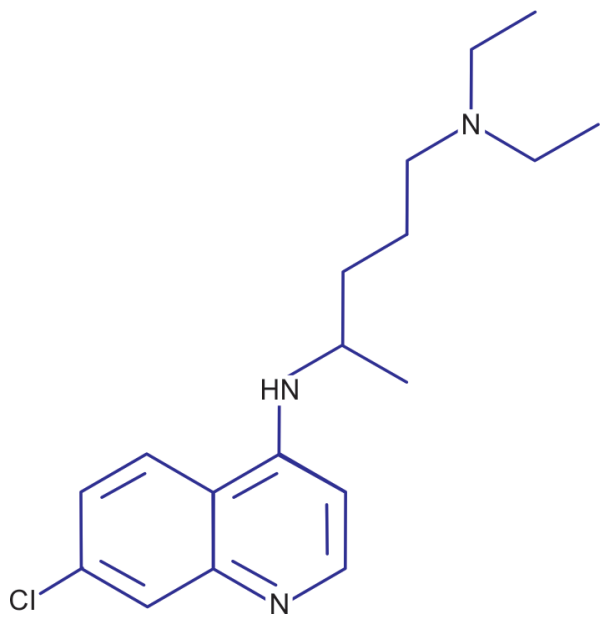

Fig. 2: Choroquine 

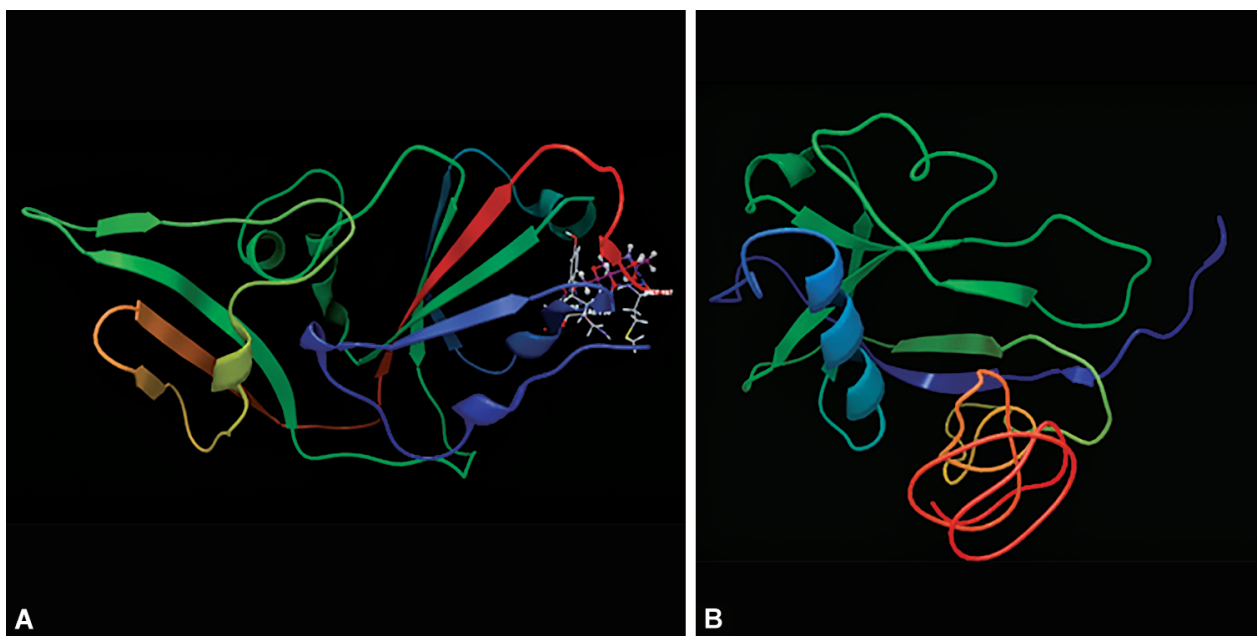

Figs 3A and B: PDB ID:5ZXR BATCOV. Novel coronavirus 2019 (QHD43415)-I-TASSER

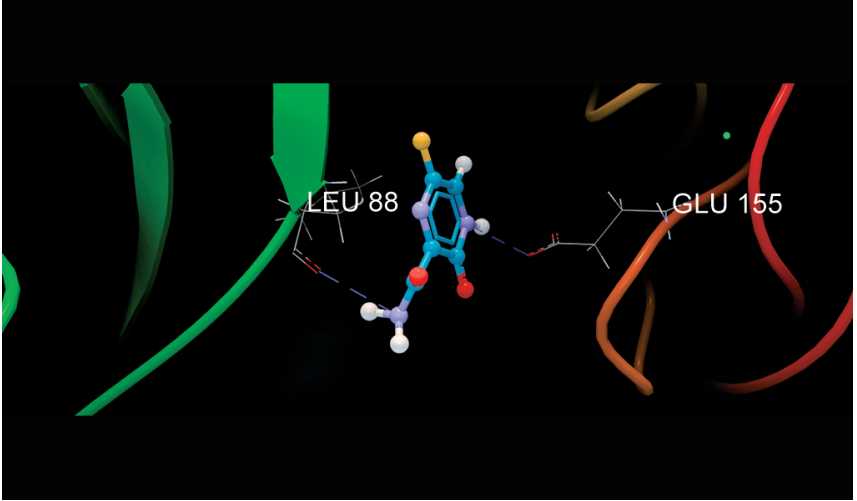

Fig. 4: Favipiravir in binding pocket

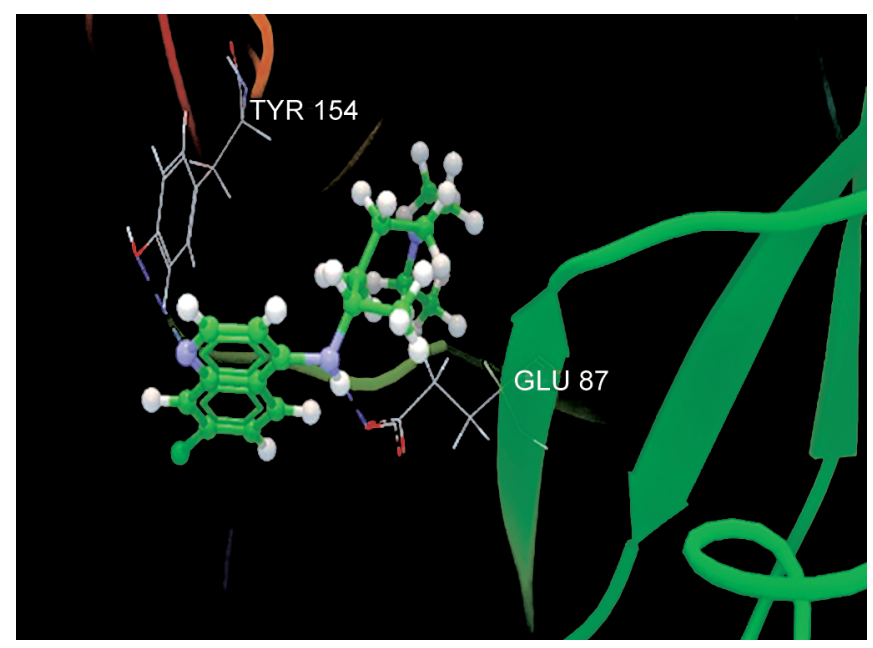

Fig. 6: Chloroquine with interaction TYR154 and GLU87

The protein of topoisomerase-2 with PDB ID:2ZXM was retrieved into the workbench from RCB:PDB. All the ligands were docked into the active site ATPase domain/motif of 1ZXM, validation of the active site was done by re-docking with ATP and ANP onto the active site. The RMSD index of binding topology between the original ligand and re-docked ligand onto the active site is 0.025 (Fig. 13). ${ }^{7-9}$

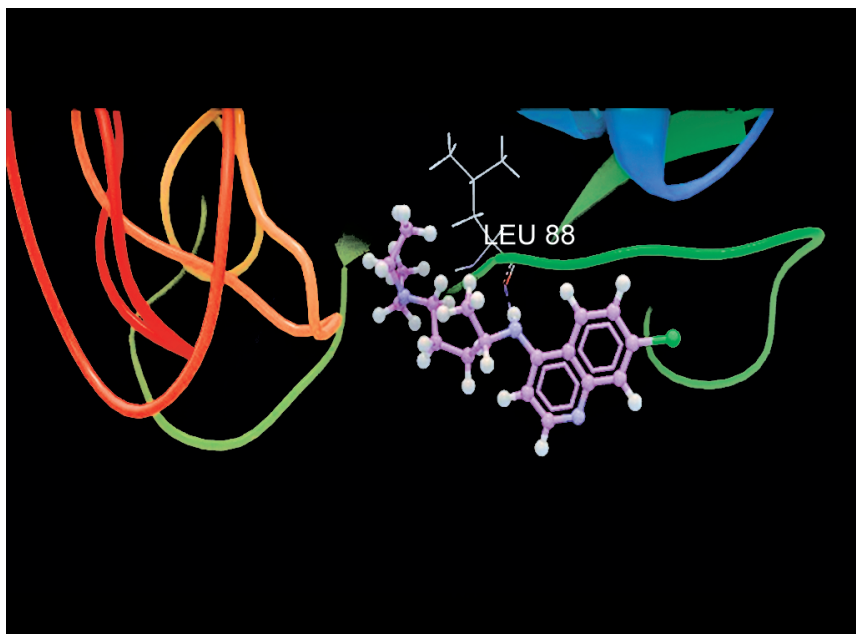

Fig. 5: Chloroquine with interaction on LEU88

\begin{tabular}{lll}
\hline Ligands & $\begin{array}{l}\text { Interacting residues } \\
\text { within } 6 A^{\circ}\end{array}$ & $\begin{array}{l}\text { Hydrophobic } \\
\text { interactions }\end{array}$ \\
\hline $\begin{array}{l}\text { Co-crystallized } \\
\text { ATPase }\end{array}$ & $\begin{array}{l}\text { Asn120, Asp94, Arg162 } \\
\text { Gln376, Gly164, Gly168, } \\
\text { Tyr165, Lys168, and }\end{array}$ & $\begin{array}{l}\text { Ser149, Tyr165, and } \\
\text { Gly-166 }\end{array}$ \\
& $\begin{array}{l}\text { Asn91 } \\
\text { KAL-series }\end{array}$ & $\begin{array}{l}\text { Ser149, Tyr165, and } \\
\text { Gly-166 }\end{array}$ \\
& & $\begin{array}{l}\text { Asn120, Asp94, } \\
\text { Arg162 Gln376 Gly164, } \\
\text { Gly168, Tyr165, Lys168, } \\
\text { and Asn91 }\end{array}$ \\
\hline
\end{tabular}

Immunocompromised Patients Treatment Regimen

Coronavirus infects all the population irrespective of age/sex. However, the effects or symptoms of the coronavirus are evident only in certain populations who are immunocompromised and adverse effects are observed only in those populations who were underlying manifestations of heart failure, renal infection, acute immune deficiency, and tuberculosis. As the coronavirus targets the ACE enzyme protein in the human body. The usage of ACE inhibitors for congestive heart failure (CHF) patients to reduce the 


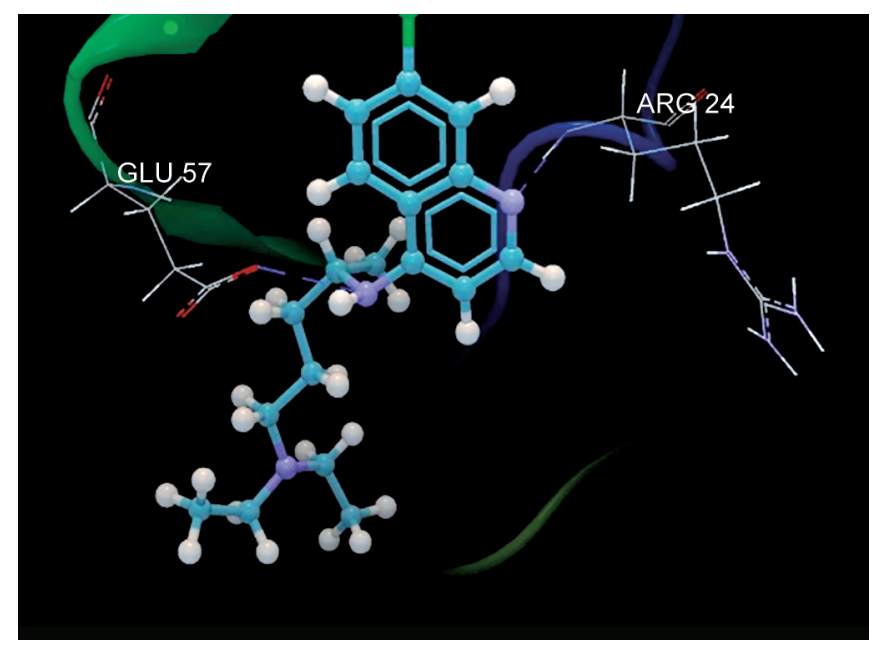

Fig. 7: Chloroquine interaction with GLU57 and ARG24

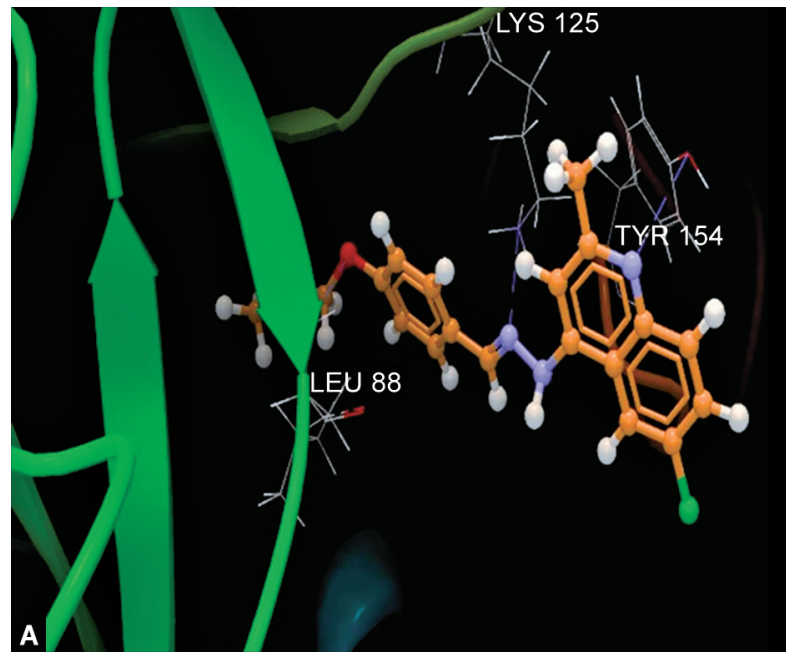

Figs 9A and B: (A) KAL-03 interaction with TYR154, LYS125, and LEU88. (B)

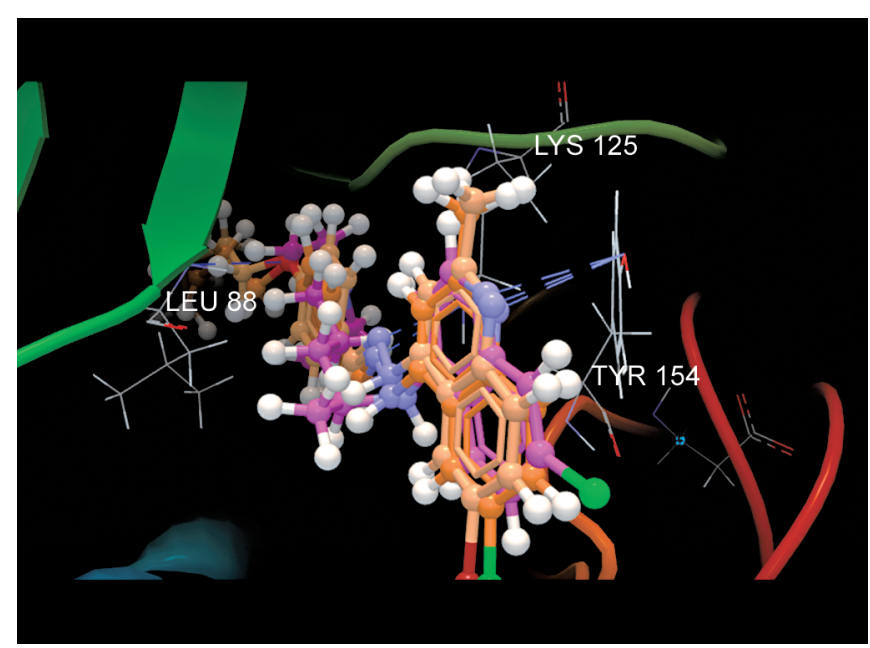

Fig. 10: Overlaid poses of chloroquine: quinolone-based hydrazones from our lab in the active site nCoV-19

vasoconstriction with renin-angiotensin system of management of hypertension will further aggravate the exposure of coronavirus as

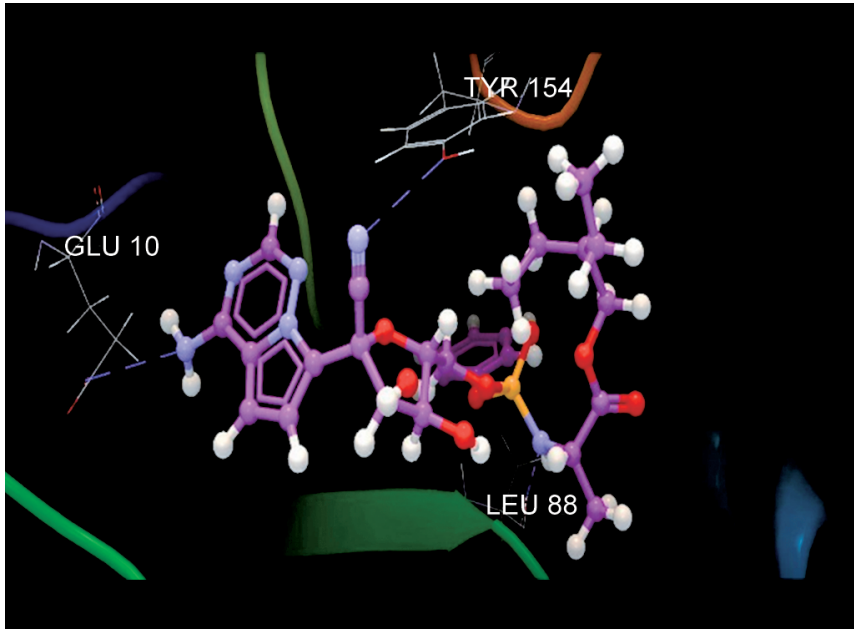

Fig. 8: Remdesivir interaction with TYR154, GLU10, and LEU88

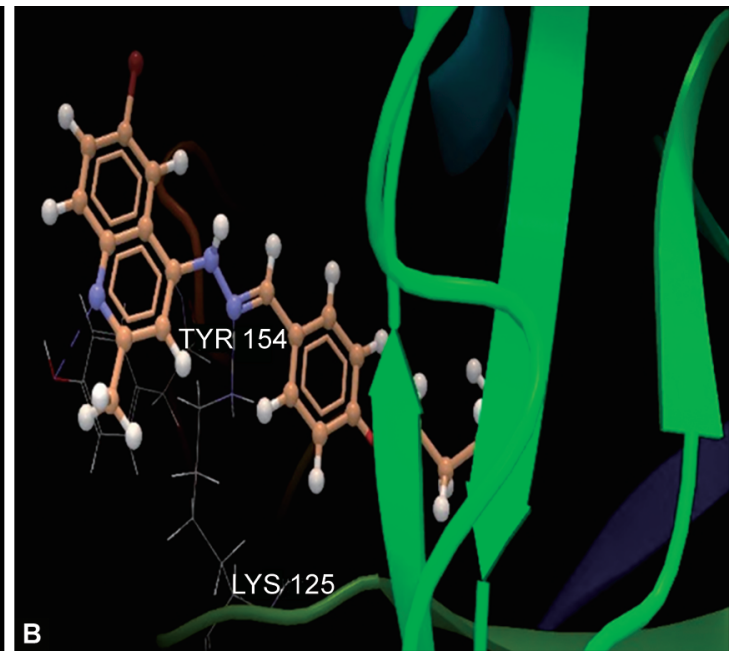

KAL-06 interaction with TYR154 and LYS125

the ACE isoforms are the increased substrate access to coronavirus attack. Dry cough is the major clinical manifestation probably due to inhibition or binding to ACE or increased bradykinins and inflammatory mediators. Genetic predisposition factor of effect of coronavirus, it is reported the patents based on the mortality rate the $\mathrm{A}+$ and $\mathrm{A}$ blood group the fatal effects of corona are more in comparison with $\mathrm{O}+$ and $\mathrm{O}$ blood group patients. Usage of paracetamol for inflammation and antipyretic effect is advised over ibuprofen as clinically ibuprofen alleviates the risk of increased effect of the corona. Further Actemra which is under drug pipeline for the treatment of inflammation (rheumatoid) is further being tested in patients with corona. The drug-likeness index and toxicity profiling using the faf webserver clearly demarcate the toxicity index of molecules however their safety was also screened on healthy cell lines. ${ }^{10-13}$

\section{Conclusion}

The active site modeling of modeled protein of COVID-19 available by I-TASSER using binding pocket search protocol of CLC drug Discovery workbench4.0. Further, the binding pockets were docked with all the retroviral drugs and chloroquine to ascertain binding efficiency; however, the binding pocket did 


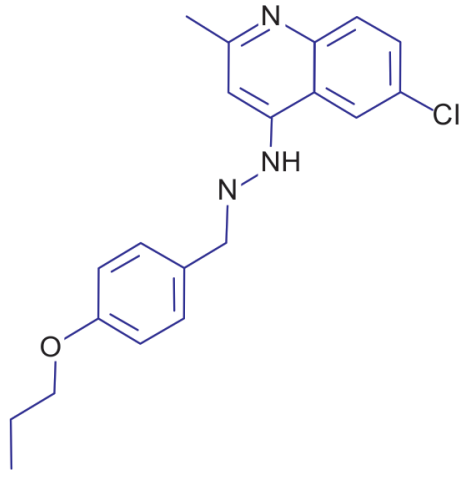

Molecular formula: $\mathrm{C} 20 \mathrm{H} 20 \mathrm{Cl} \mathrm{N} 3 \mathrm{O}$

Molecular weight: 353.13

Number of HBA: 3

Number of HBD: 1

MolLogP: $6.27(>5)$

MolLogS: -6.60 (in Log(moles/L)) 0.09 (in mg/L)

MoIPSA: $36.23 \mathrm{~A}^{2}$

MolVol: $343.08 \mathrm{~A}^{3}$

Number of stereo centers: 0

Fig. 11: Molecular properties of designed ligands

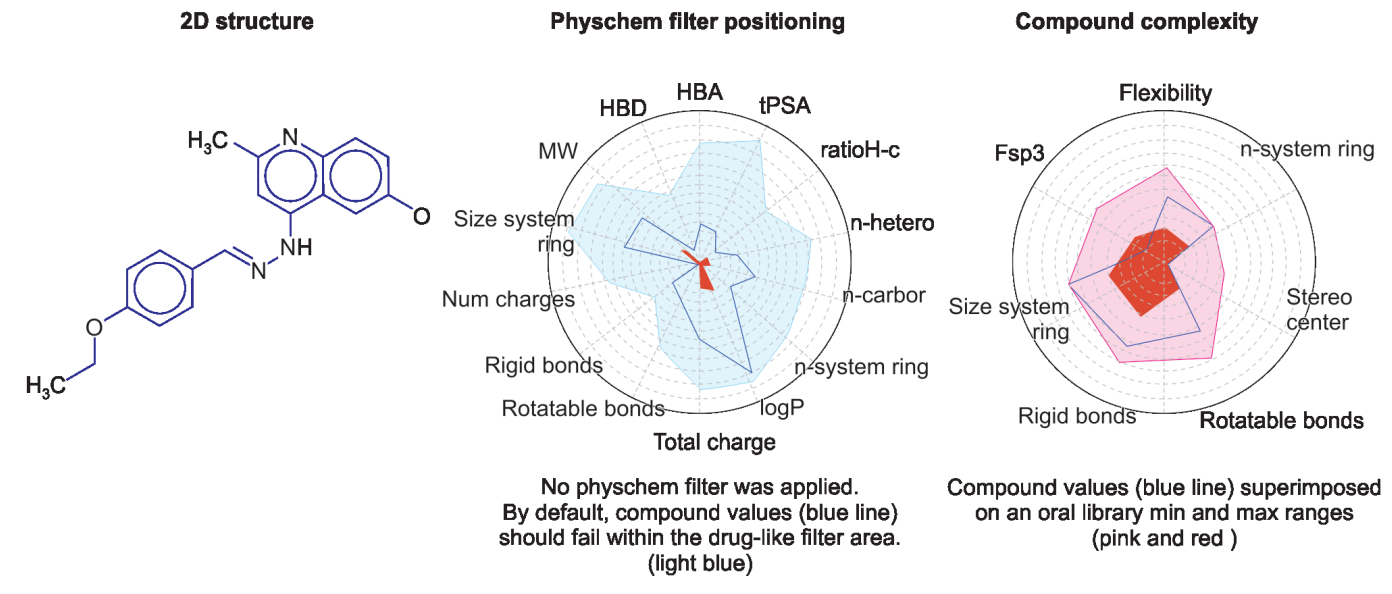

Golden triangle rule

Oral property space

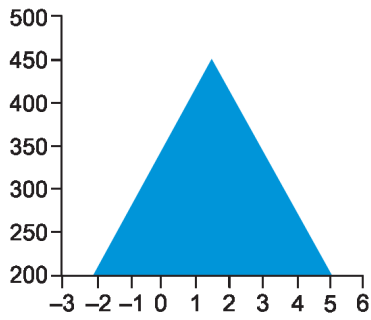

Compounds located in the yellow triangle are likely to have an optimal permeability (low clearance) and a good metabolic stability Johnson et al-Bioorg. Med. Chem. Lett. 2009

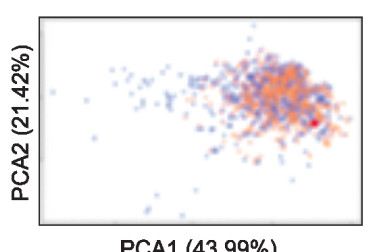

PCA1 (43.99\%)
Oral absorption estimation

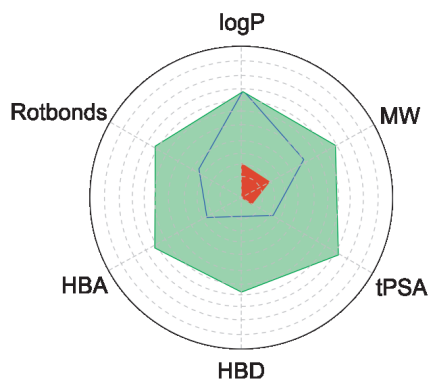

Compound values (blue line) should fall withing RO5 and Veber rules area. (light green) chemical property space was obtained principal physicochemical descriptors of

(the user's compound) compared to two

oral libraries extracted from eDrugs and Drugbank. See the correlation graph

Pfizer $3 / 75$ rule positioning

\section{Oral bioavailability}

Lipinski RO5

Vever rule

Egan rule

Bayer oral physchem score

\section{Drug safety profiling}

GSK 4/400 rule

Pfizer $3 / 75$ rule

Phospholipidosis non induce

Lilly medChem rules: Not computed

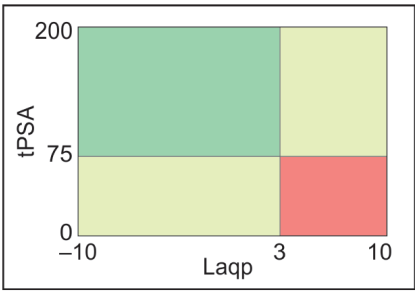

Compounds located in the red square are likely to cause toxicity and experimental promiscuity

Fig. 12: ADMET analysis of designed ligands 

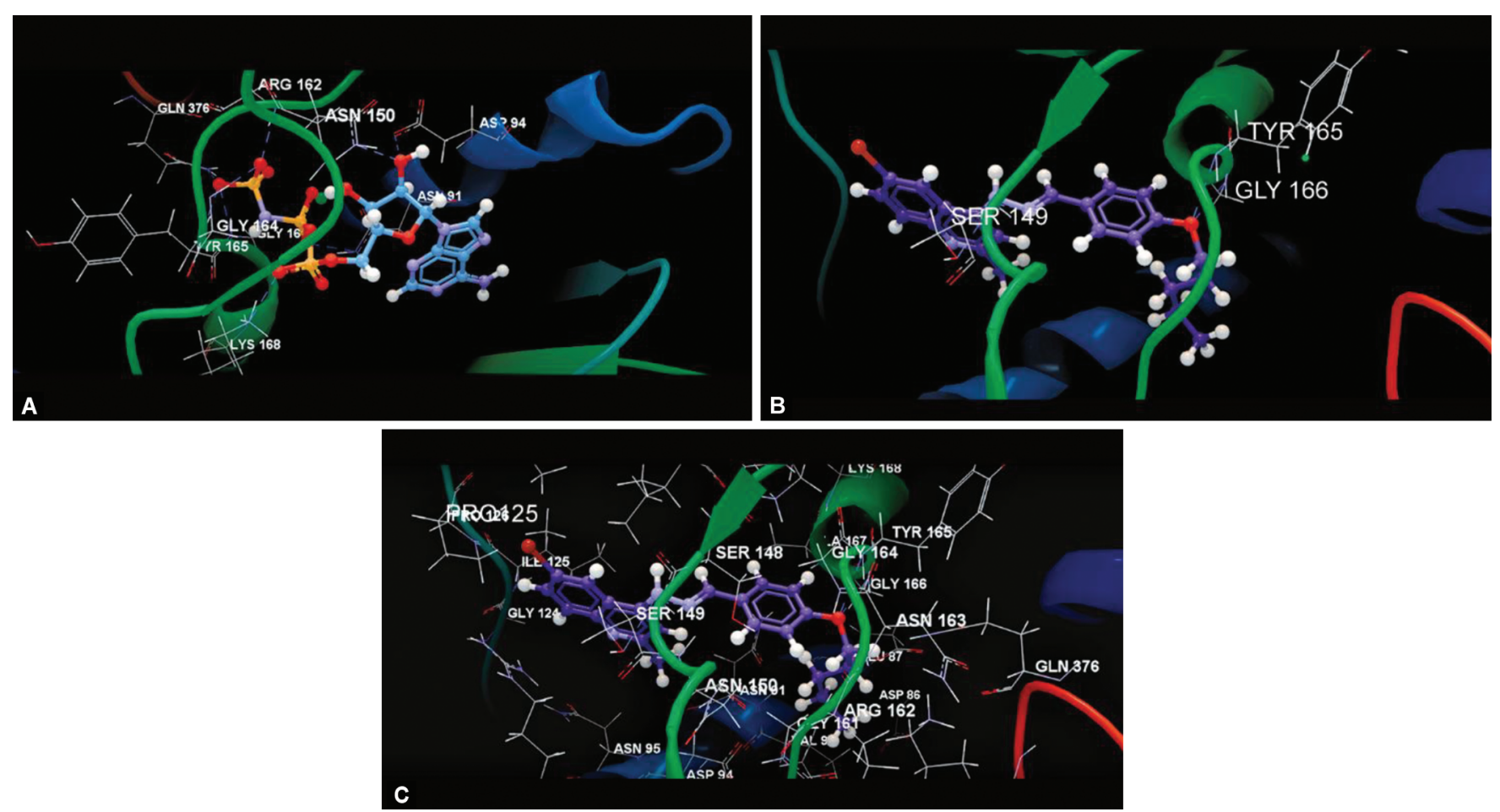

Figs $13 \mathrm{~A}$ to $\mathrm{C}$ : (A) Validation of binding pocket re-docking of ANP into the active site; (B) Representative hydrogen-bonding interaction of KAL-08 on ATP-binding site; (C) Hydrogen bonding and hydrophobic interaction of KAL-08 on ATPase-binding site of 1ZXM

not explicit any interactions with approved ACE inhibitors. Though the phylogenetic similarity between bat coronavirus and human coronavirus the active site prediction suggests that the two proteins have different binding sites. Our molecule which was recently published for topoisomerase-1 inhibitors was also docked onto the predicted active site along with chloroquine using DYLOMMS as principle where active site overlap of chloroquine and our reported molecules are shown similarity in binding sites in addition to promising topoisomerase-II binding. As the previously reported quinoline analogs from our lab are promising anticancer agents further computational approach of target site-directed docking for their potential treatment of malaria and viral infection and the in vitro and in vivo screening of molecules are underway.

\section{References}

1. Paraskevis D, Kostaki EG, Magiorkinis G, et al. Full-genome evolutionary analysis of the novel corona virus (2019-NCoV) rejects the hypothesis of emergence as a result of a recent recombination event. Infect Genet Evol 2020;79:104212. DOI: 10.1016/j.meegid.2020. 104212.

2. Discovery of a novel coronavirus associated with the recent pneumonia outbreak in humans and its potential bat origin | bioRxiv https://www.biorxiv.org/content/10.1101/2020.01.22.914952v2 (accessed Apr 20, 2021).

3. Zhou N, Pan T, Zhang J, et al. Glycopeptide antibiotics potently inhibit Cathepsin L in the late endosome/lysosome and block the entry of Ebola virus, middle east respiratory syndrome coronavirus (MERS$\mathrm{CoV}$ ), and severe acute respiratory syndrome coronavirus (SARS-CoV). J Biolog Chem 2016. 9218-9232. DOI: 10.1074/jbc.M116.716100.
4. Gordon CJ, Tchesnokov EP, Feng JY, et al. The antiviral compound remdesivir potently inhibits RNA-dependent RNA polymerase from MERS corona virus. J Biolog Chem 2020;295(15):4773-4779. DOI: 10.1074/jbc.AC120.013056.

5. Wang M, Cao R, Zhang L, et al. Remdesivir and chloroquine effectively inhibit the recently emerged novel coronavirus (2019-nCoV) in vitro. Cell Res 2020;30:269-271. DOI: 10.1038/s41422-020-0282-0.

6. Cheng VCC, Lau SKP, Woo PCY, et al. Severe acute respiratory syndrome coronavirus as an agent of emerging and reemerging infection. Yuen Clin Microbiol Rev 2007;20(4):660-694.

7. Wu C, Liu Y, Yang Y, et al. Analysis of therapeutic targets for SARSCoV-2 and discovery of potential drugs by computational methods. Acta Pharm Sin B 2020;10(5):766-788. DOI: 10.1016/j.apsb.2020.02.008.

8. Joshi G, Amrutkar SM, Baviskar AT, et al. Synthesis and biological evaluation of new 2, 5 dimethylthiophene/furan based $\mathrm{N}$-acetyl pyrazolines as selective topoisomerase II inhibitors Darpan. RSC Adv. 2016;6:14880-14892.

9. Roy A, Kucukural A, Zhang Y. I-TASSER: a unified platform for automated protein structure and function prediction. Nat Proto 2010;5:725-738. DOI: 10.1038/nprot.2010.5.

10. Yang J, Yan R, Roy A, et al. The I-TASSER suite: protein structure and function prediction. Nat Metho 2015;12:7-8. DOI: 10.1038/ nmeth.3213.

11. Yang J, Zhang Y. I-TASSER server: new development for protein structure and function predictions. Nucleic Acids Res 2015;43:W174W181.

12. Lagorce D, Sperandio O, Galons H, et al. FAF-Drugs2: free ADME/tox filtering tool to assist drug discovery and chemical biology projects. BMC Bioinformatics 2008;9:396. DOI: 10.1186/1471-2105-9-396.

13. Katariya KD, Shah SR, Reddy D. Anticancer, antimicrobial activities of quinoline based hydrazone analogues: synthesis, characterization and molecular docking. Bioorga Chem 2020;94:103406. DOI: 10.1016/j. bioorg.2019.103406ISSN 0045-2068. 